CORRECTION

\title{
Correction: Solving unsolved rare neurological diseases-a Solve-RD viewpoint
}

Rebecca Schüle, Dagmar Timmann, Corrie E. Erasmus, Jennifer Reichbauer, Melanie Wayand, Solve-RD-DITF-RND, Bart van de Warrenburg, Ludger Schöls, Carlo Wilke (iD), Andrea Bevot, Stephan Zuchner, Sergi Beltran (iD, Steven Laurie (iD), Leslie Matalonga, Holm Graessner (D), Matthis Synofzik (D) and The Solve-RD Consortium

(c) The Author(s), under exclusive licence to European Society of Human Genetics 2021

European Journal of Human Genetics (2021) 29:1462-1465; https://doi.org/10.1038/s41431-021-00935-5

Correction to: European Journal of Human Genetics https://doi.org/ 10.1038/s41431-021-00901-1, published online 10 May 2021

In the original publication of the article, consortium author lists were missing in the article. The details are given below:

\section{Solve-RD-DITF-RND}

Jonathan Baets ${ }^{13,14,15}$, Peter Balicza ${ }^{16}$, Patrick Chinnery ${ }^{17}$, Alexandra Dürr ${ }^{18,19,20}$, Tobias Haack ${ }^{12}$, Holger Hengel ${ }^{2,21}$, Rita Horvath ${ }^{22}$, Henry Houlden ${ }^{23}$, Erik-Jan Kamsteeg ${ }^{24}$, Christoph Kamsteeg ${ }^{24}$, Katja Lohmann ${ }^{25}$, Alfons Macaya ${ }^{26}$, Anna Marcé-Grau ${ }^{26}$, Ales Maver $^{27}$, Judit Molnar ${ }^{16}$, Alexander Münchau ${ }^{25}$, Borut Peterlin ${ }^{27}$, Olaf Riess ${ }^{12,28}$, Ludger Schöls ${ }^{2,21}$, Rebecca Schüle ${ }^{2,21}$, Giovanni Stevanin ${ }^{18,19,20,29,30}$, Matthis Synofzik ${ }^{2,21}$, Vincent Timmerman ${ }^{31,32}$, Bart van de Warrenburg ${ }^{33}$, Nienke van $\mathrm{Os}^{33,34}$, Jana Vandrovcova $^{23}$, Melanie Wayand ${ }^{2,21}$, Carlo Wilke ${ }^{2,21}$

${ }^{13}$ Peripheral Neuropathy Research Group, University of Antwerp, Antwerp, Belgium

${ }^{14}$ Neuromuscular Reference Centre, Department of Neurology, Antwerp University Hospital, Antwerpen, Belgium

${ }^{15}$ Laboratory of Neuromuscular Pathology, Institute Born-Bunge, University of Antwerp, Antwerpen, Belgium

${ }^{16}$ Semelweis University Budapest, Budapest, Hungary

${ }^{17}$ Center for Hereditary Tumor Syndromes, University Hospital Bonn, Bonn, Germany

${ }^{18}$ Institut National de la Santé et de la Recherche Medicale (INSERM) U1127, Paris, France

${ }^{19}$ Centre National de la Recherche Scientifique, Unité Mixte de Recherche (UMR), Paris, France

${ }^{20}$ Unité Mixte de Recherche en Santé 1127, Université Pierre et Marie Curie (Paris 06), Sorbonne Universités, Paris, France

${ }^{21}$ Department of Neurodegeneration, Hertie Institute for Clinical Brain Research $(\mathrm{HIH})$, University of Tübingen, Tübingen, Germany
${ }^{22}$ University of Cambridge, England, UK

${ }^{23}$ Department of Neuromuscular Diseases, UCL Queen Square Institute of Neurology and The National Hospital for Neurology and Neurosurgery, London, UK

${ }^{24}$ Department of Human Genetics, Radboud University Medical Center, Nijmegen, The Netherlands

${ }^{25}$ University of Lübeck, Lübeck, Germany

${ }^{26}$ Hospital Vall d'Hebron, Barcelona, Spain

${ }^{27}$ University of Ljubljana, Ljubljana, Slovenia

${ }^{28}$ Centre for Rare Diseases, University of Tübingen, Tübingen, Germany

${ }^{29}$ Institut du Cerveau-ICM, Paris, France

${ }^{30}$ Ecole Pratique des Hautes Etudes, Paris Sciences et Lettres Research University, Paris, France

${ }^{31}$ Peripheral Neuropathy Research Group, Department of Biomedical Sciences, University of Antwerp, Antwerp, Belgium

${ }^{32}$ Institute Born Bunge, Antwerp, Belgium

${ }^{33}$ Donders Institute for Brain, Cognition and Behaviour, Radboud University Medical Center, Nijmegen, The Netherlands

${ }^{34}$ Department of Neurology, Radboud University Medical Center, Nijmegen, The Netherlands

\section{The Solve-RD Consortium}

Olaf Riess ${ }^{12,28}$, Tobias B. Haack ${ }^{12}$, Holm Graessner ${ }^{12,28}$, Birte Zurek $^{12,28}$, Kornelia Ellwanger ${ }^{12,28}$, Stephan Ossowski ${ }^{12}$, German Demidov $^{12}$, Marc Sturm ${ }^{12}$, Julia M. Schulze-Hentrich ${ }^{12}$, Rebecca Schüle ${ }^{2,21}$ ' Christoph Kessler ${ }^{2,21}$, Melanie Wayand ${ }^{2,21}$, Matthis Synofzik 2,21 , Carlo Wilke 2,21 , Andreas Traschütz ${ }^{2,21}$, Ludger Schöls $^{2,21}$, Holger Hengel ${ }^{2,21}$, Peter Heutink ${ }^{2,21}$, Han Brunner 24,33,35, Hans Scheffer 24,35 , Nicoline Hoogerbrugge ${ }^{24,36}$, Alexander' 
Hoischen $24,36,37$, Peter A. C. 't Hoen ${ }^{36,38}$, Lisenka E. L. M. Vissers ${ }^{24,33}$, Christian Gilissen ${ }^{24,36}$, Wouter Steyaert ${ }^{24,36}$, Karolis Sablauskas ${ }^{24}$, Richarda M. de Voer ${ }^{24,36}$, Erik-Jan Kamsteeg ${ }^{24}$, Bart van de Warrenburg ${ }^{33,34}$, Nienke van $\mathrm{Os}^{33,34}$, Iris te Paske 24,36 , Erik Janssen ${ }^{24,36}$, Elke de Boer ${ }^{24,33}$, Marloes Steehouwer ${ }^{24}$, Burcu Yaldiz $^{24}$, Tjitske Kleefstra ${ }^{24,33}$, Anthony J. Brookes ${ }^{39}$, Colin Veal ${ }^{39}$, Spencer Gibson ${ }^{39}$, Marc Wadsley ${ }^{39}$, Mehdi Mehtarizadeh ${ }^{39}$, Umar Riaz $^{39}$, Greg Warren ${ }^{39}$, Farid Yavari Dizjikan ${ }^{39}$, Thomas Shorter ${ }^{39}$, Ana Töpf ${ }^{40}$, Volker Straub ${ }^{40}$, Chiara Marini Bettolo ${ }^{40}$, Sabine Specht ${ }^{40}$, Jill Clayton-Smith ${ }^{4}{ }^{\prime}$, Siddharth Banka ${ }^{41,42}$, Elizabeth Alexander $^{41}$, Adam Jackson ${ }^{41}$, Laurence Faivre ${ }^{43,44,45,46,47}$, Christel Thauvin ${ }^{44,45,46,47}$, Antonio Vitobello ${ }^{45}$, Anne-Sophie DenomméPichon $^{45}$, Yannis Duffourd ${ }^{45,46}$, Emilie Tisserant ${ }^{45}$, Ange-Line Bruel $^{45}$, Christine Peyron ${ }^{48,49}$, Aurore Pélissier ${ }^{49}$, Sergi Beltran ${ }^{9,10}$, Ivo Glynne Gut ${ }^{10}$, Steven Laurie ${ }^{10}$, Davide Piscia ${ }^{10}$, Leslie Matalonga $^{10}$, Anastasios Papakonstantinou ${ }^{10}$, Gemma Bullich ${ }^{10}$, Alberto Corvo ${ }^{10}$, Carles Garcia ${ }^{10}$, Marcos Fernandez-Callejo ${ }^{10}$, Carles Hernández ${ }^{10}$, Daniel Picó ${ }^{10}$, Ida Paramonov ${ }^{10}$, Hanns Lochmüller $^{10}$, Gulcin Gumus ${ }^{50}$, Virginie Bros-Facer ${ }^{51}$, Ana Rath ${ }^{52}$, Marc Hanauer ${ }^{52}$, Annie Olry ${ }^{52}$, David Lagorce ${ }^{52}$, Svitlana Havrylenko ${ }^{52}$ Katia Izem ${ }^{52}$, Fanny Rigour ${ }^{52}$, Giovanni Steva$\operatorname{nin}^{18,19,20,29,30} \quad$ Alexandra Durr ${ }^{19,20,29,53}$ Claire-Sophie Davoine ${ }^{19,20,29,30}$, Léna Guillot-Noel ${ }^{19,20,29,30}$, Anna Heinzmann ${ }^{19,20,29,54}$, Giulia Coarelli ${ }^{19,20,29,54}$, Gisèle Bonne ${ }^{55}$, Teresinha Evangelista ${ }^{55}$,' Valérie Allamand ${ }^{55}$, Isabelle Nelson ${ }^{55}$, Rabah Ben Yaou ${ }^{5,56,57}$, Corinne Metay ${ }^{55,58}$, Bruno Eymard ${ }^{55,56}$, Enzo Cohen ${ }^{55}$, Antonio Atalaia ${ }^{55}$, Tanya Stojkovic ${ }^{55,56}$, Milan Macek Jr. ${ }^{59}$, Marek Turnovec ${ }^{59}$, Dana Thomasová59, Radka Pourová Kremliková' ${ }^{59}$, Vera Frankovás9', Markéta Havlovicová ${ }^{59}$, Vlastimil Kremlik ${ }^{59}$, Helen Parkinson $^{60}$, Thomas Keane ${ }^{60}$, Dylan 'Spalding ${ }^{60}$, Alexander Senf ${ }^{60}{ }^{\prime}$ Peter Robinson ${ }^{61}$, Daniel Danis ${ }^{61}$, Glenn Robert ${ }^{62}$, Alessia Costa ${ }^{62}$, Christine Patch ${ }^{62,63}$, Mike Hanna ${ }^{64}$, Henry Houlden ${ }^{65}$, Mary Reilly ${ }^{64}{ }^{\prime}$ Jana Vandrovcova ${ }^{65}$, Francesco Muntoni ${ }^{66,67}$, Irina Zaharieva ${ }^{66^{\prime}}$ Anna Sarkozy ${ }^{66}$, Vincent Timmerman ${ }^{31,32}$, Jonathan Baets ${ }^{13,14,15^{\prime}}$, Liedewei Van de Vondel ${ }^{13,32}$, Danique Beijer ${ }^{13,32}$, Peter de Jonghe ${ }^{14,32}$, Vincenzo Nigro ${ }^{68,69}$, Sandro Banfi ${ }^{68,69}$ ', Annalaura Torella $^{68}$, Francesco Musacchia ${ }^{68,69}$, Giulio Piluso68', Alessandra Ferlini $^{70}$, Rita Selvatici ${ }^{70}$, Rachele Rossi ${ }^{70}$, Marcella Neri ${ }^{70}$, Stefan Aretz $^{17,7_{1}}$, Isabel Spier ${ }^{17,71}$ Anna Katharina Sommer ${ }^{71}$, Sophia Peters $^{71}$, Carla Oliveira ${ }^{72,73,74}$, Jose Garcia Pelaez ${ }^{72,73}$, Ana Rita Matos $^{72,73}$, Celina São Joséé ${ }^{72,73}$, Marta Ferreira ${ }^{72,73}$, Irene Gullo ${ }^{72,73,74}$, Susana Fernandes ${ }^{72,75}$, Luzia Garrido ${ }^{76}$, Pedro Ferreira $^{72,73,77}$, Fátima Carneiro ${ }^{72,73,74}$, Morris A. Swertz ${ }^{78}$, Lennart Johansson $^{78}$, Joeri K. van der Velde ${ }^{78}$, Gerben van der Vries ${ }^{78}$, Pieter B. Neerincx ${ }^{78}$, Dieuwke Roelofs-Prins ${ }^{78}$, Sebastian Köhler ${ }^{79}$, Alison Metcalfe ${ }^{62,80}$, Alain Verloes ${ }^{81,82}$, Séverine Drunat ${ }^{81,82}$, Caroline Rooryck ${ }^{83}$, Aurelien Trimouille ${ }^{84}$, Raffaele Castello ${ }^{69}$, Manuela Morleo $^{69}$, Michele Pinelli ${ }^{69}$, Alessandra Varavallo ${ }^{69}$, Manuel Posada De la Paz ${ }^{85}$, Eva Bermejo Sánchez ${ }^{85}$, Estrella López Martín ${ }^{85}$, Beatriz Martínez Delgado ${ }^{85}$, F. Javier Alonso García de la Rosa ${ }^{85}$, Andrea Ciolf ${ }^{86}$, Bruno Dallapiccola ${ }^{86}$, Simone Pizzi ${ }^{86}$, Francescá Clementina Radio ${ }^{86}$, Marco Tartaglia ${ }^{86}$, Alessandra Renieri ${ }^{87,88,89}$ Elisa Benetti ${ }^{87}$, Peter Balicza ${ }^{90}$, Maria Judit Molnar ${ }^{90}$, Ales Maver ${ }^{91}{ }^{\prime}$ Borut Peterlin ${ }^{91}$, Alexander Münchau ${ }^{92}$, Katja Lohmann ${ }^{92}$, Rebeccá Herzog ${ }^{92}$, Martje Pauly ${ }^{92}$, Alfons Macaya ${ }^{93}$, Anna Marcé-Grau ${ }^{93}$, Andres Nascimiento Osorio ${ }^{94}$, Daniel Natera de Benito ${ }^{94}$, Hann' Lochmüller $^{95,96,97}$, Rachel Thompson ${ }^{95,97}$, Kiran Polavarapu ${ }^{95}$, David Beeson ${ }^{98}$,' Judith Cossins ${ }^{98}$, Pedro $M$. Rodriguez Cruz ${ }^{98}$, Peter Hackman'99, Mridul Johari'99, Marco Savarese ${ }^{99}$, Bjarné Udd $^{99,100,101}$, Rita 'Horvath ${ }^{102}$, Gabriel Capella ${ }^{103}$, Laura 'Valle ${ }^{103}$, Elke Holinski-Feder ${ }^{104}$, Andreas Laner ${ }^{104}$, Verena Steinke-Lange ${ }^{104}$, Evelin Schröck ${ }^{105}$, Andreas Rump ${ }^{105,106}$

\footnotetext{
${ }^{35}$ Department of Clinical Genetics, Maastricht University Medical Centre, Maastricht, The Netherlands
}

${ }^{36}$ Radboud Institute for Molecular Life Sciences, Nijmegen, The Netherlands

${ }^{37}$ Department of Internal Medicine and Radboud Center for Infectious Diseases (RCl), Radboud University Medical Center, Nijmegen, The Netherlands

${ }^{38}$ Center for Molecular and Biomolecular Informatics, Radboud University Medical Center, Nijmegen, The Netherlands

${ }^{39}$ Department of Genetics and Genome Biology, University of Leicester, Leicester, UK

${ }^{40}$ John Walton Muscular Dystrophy Research Centre, Translational and Clinical Research Institute, Newcastle University and Newcastle Hospitals NHS Foundation Trust, Newcastle upon Tyne, UK

${ }^{41}$ Division of Evolution and Genomic Sciences, School of Biological Sciences, Faculty of Biology, Medicine and Health, University of Manchester, Manchester, UK

${ }^{42}$ Manchester Centre for Genomic Medicine, St Mary's Hospital, Manchester University Hospitals NHS Foundation Trust, Health Innovation Manchester, Manchester, UK

${ }^{43}$ Dijon University Hospital, Genetics Department, Dijon, France

${ }^{44}$ Dijon University Hospital, Centre of Reference for Rare Diseases: Development disorders and malformation syndromes, Dijon, France

${ }^{45}$ Inserm - University of Burgundy-Franche Comté UMR1231 GAD, Dijon, France

${ }^{46}$ Dijon University Hospital, FHU-TRANSLAD, Dijon, France

${ }^{47}$ Dijon University Hospital, GIMI Institute, Dijon, France

${ }^{48}$ University of Burgundy-Franche Comté, Dijon Economics Laboratory, Dijon, France

${ }^{49}$ University of Burgundy-Franche Comté, FHU-TRANSLAD, Dijon, France

${ }^{50}$ EURORDIS-Rare Diseases Europe, Sant Antoni Maria Claret 167, Barcelona, Spain

${ }^{51}$ EURORDIS-Rare Diseases Europe, Plateforme Maladies Rares, Paris, France

${ }^{52}$ INSERM, US14 - Orphanet, Plateforme Maladies Rares, Paris, France

${ }^{53}$ Centre de Référence de Neurogénétique, Hôpital de la PitiéSalpêtrière, Assistance Publique-Hôpitaux de Paris (AP-HP), Paris, France

${ }^{54}$ Hôpital de la Pitié-Salpêtrière, Assistance Publique-Hôpitaux de Paris (AP-HP), Paris, France

${ }^{55}$ Sorbonne Université, INSERM UMRS_974, Center of Research in Myology, Paris, France

${ }^{56} \mathrm{AP}-\mathrm{HP}$, Centre de Référence de Pathologie Neuromusculaire Nord, Est, Ile-de-France, Institut de Myologie, G.H. Pitié- Salpêtrière, Paris, France 
1464

${ }^{57}$ Institut de Myologie, Equipe Bases de données, G.H. PitiéSalpêtrière, Paris, France

${ }^{58} \mathrm{AP}-\mathrm{HP}$, Unité Fonctionnelle de Cardiogénétique et Myogénétique Moléculaire et Cellulaire, G.H. Pitié-Salpêtrière, Paris, France

${ }^{59}$ Department of Biology and Medical Genetics, Charles University Prague-2nd Faculty of Medicine and University Hospital Motol, Prague, Czech Republic

${ }^{60}$ European Bioinformatics Institute, European Molecular Biology Laboratory, Wellcome Genome Campus, Hinxton, Cambridge, UK

${ }^{61}$ Jackson Laboratory for Genomic Medicine, Farmington, CT, USA

${ }^{62}$ Florence Nightingale Faculty of Nursing and Midwifery, King's College, London, UK

${ }^{63}$ Genetic Counselling, Genomics England, Queen Mary University of London, Dawson Hall, London, UK

${ }^{64}$ MRC Centre for Neuromuscular Diseases and National Hospital for Neurology and Neurosurgery, UCL Queen Square Institute of Neurology, London, UK

${ }^{65}$ Department of Neuromuscular Diseases, UCL Queen Square Institute of Neurology, London, UK

${ }^{66}$ Dubowitz Neuromuscular Centre, UCL Great Ormond Street Hospital, London, UK

${ }^{67}$ NIHR Great Ormond Street Hospital Biomedical Research Centre, London, UK

${ }^{68}$ Dipartimento di Medicina di Precisione, Università degli Studi della Campania "Luigi Vanvitelli", Napoli, Italy

${ }^{69}$ Telethon Institute of Genetics and Medicine, Pozzuoli, Italy

${ }^{70}$ Unit of Medical Genetics, Department of Medical Sciences, University of Ferrara, Ferrara, Italy

${ }^{71}$ Institute of Human Genetics, University of Bonn, Bonn, Germany

${ }^{72}$ i3S - Instituto de Investigação e Inovação em Saúde, Universidade do Porto, Porto, Portugal

${ }^{73}$ IPATIMUP - Institute of Molecular Pathology and Immunology of the University of Porto, Porto, Portugal

${ }^{74}$ Department of Pathology, Faculty of Medicine, University of Porto, Porto, Portugal

${ }^{75}$ Department of Genetics, Faculty of Medicine, University of Porto, Porto, Portugal

${ }^{76}$ CHUSJ, Centro Hospitalar e Universitário de São João, Porto, Portugal

${ }^{77}$ Faculty of Sciences, University of Porto, Porto, Portugal

${ }^{78}$ Department of Genetics, Genomics Coordination Center, University Medical Center Groningen, University of Groningen, Groningen, The Netherlands
${ }^{79}$ NeuroCure Cluster of Excellence, Charité Universitätsklinikum, Charitéplatz 1, Berlin, Germany

${ }^{80}$ College of Health, Well-being and Life-Sciences, Sheffield Hallam University, Sheffield, UK

${ }^{81}$ Department of Genetics, Assistance Publique-Hôpitaux de Paris Université de Paris, Robert DEBRE University Hospital, 48 bd SERURIER, Paris, France

${ }^{82}$ INSERM UMR 1141 "NeuroDiderot", Hôpital R DEBRE, Paris, France

${ }^{83}$ University of Bordeaux, MRGM INSERM U1211, CHU de Bordeaux, Service de Génétique Médicale, Bordeaux, France

${ }^{84}$ Laboratoire de Génétique Moléculaire, Service de Génétique Médicale, CHU Bordeaux - Hôpital Pellegrin, Place Amélie Raba Léon, Bordeaux Cedex, France

${ }^{85}$ Institute of Rare Diseases Research, Spanish Undiagnosed Rare Diseases Cases Program (SpainUDP) \& Undiagnosed Diseases Network International (UDNI), Instituto de Salud Carlos III, Madrid, Spain

${ }^{86}$ Genetics and Rare Diseases Research Division, Ospedale Pediatrico Bambino Gesù, IRCCS, Rome, Italy

${ }^{87}$ Med Biotech Hub and Competence Center, Department of Medical Biotechnologies, University of Siena, Siena, Italy

${ }^{88}$ Medical Genetics, University of Siena, Siena, Italy

${ }^{89}$ Genetica Medica, Azienda Ospedaliero-Universitaria Senese, Siena, Italy

${ }^{90}$ Institute of Genomic Medicine and Rare Diseases, Semmelweis University, Budapest, Hungary

${ }^{91}$ Clinical Institute of Genomic Medicine, University Medical Centre Ljubljana, Ljubljana, Slovenia

${ }^{92}$ Institute of Neurogenetics, University of Lübeck, Lübeck, Germany

${ }^{93}$ Neurology Research Group, Vall d'Hebron Research Institute, Universitat Autònoma de Barcelona, Barcelona, Spain

${ }^{94}$ Neuromuscular Disorders Unit, Department of Pediatric Neurology. Hospital Sant Joan de Déu, Barcelona, Spain

${ }^{95}$ Department of Neuropediatrics and Muscle Disorders, Medical Center, Faculty of Medicine, University of Freiburg, Freiburg, Germany

${ }^{96}$ Centro Nacional de Análisis Genómico (CNAGCRG), Center for Genomic Regulation, Barcelona Institute of Science and Technology (BIST), Barcelona, Spain

${ }^{97}$ Children's Hospital of Eastern Ontario Research Institute, University of Ottawa, Ottawa, ON, Canada

${ }^{98}$ Nuffield Department of Clinical Neurosciences, Universityof Oxford, Oxford, UK

${ }^{99}$ Folkhälsan Research Centre and Medicum, University of Helsinki, Helsinki, Finland 
${ }^{100}$ Tampere Neuromuscular Center, Tampere, Finland

${ }^{101}$ Vasa Central Hospital, Vaasa, Finland

${ }^{102}$ Department of Clinical Neurosciences, University of Cambridge, Cambridge, UK

${ }^{103}$ Bellvitge Biomedical Research Institute (IDIBELL), Barcelona, Spain
${ }^{104}$ Medical Genetics Center (MGZ), Munich, Germany

${ }^{105}$ Institute for Clinical Genetics, Faculty of Medicine Carl Gustav Carus, Technical University Dresden, Dresden, Germany

${ }^{106}$ Center for Personalized Oncology, University Hospital Carl Gustav Carus, Technical University Dresden, Dresden, Germany 